\title{
Demonstration of ultra-high-Q small mode volume toroid microcavities on a chip
}

\author{
T. J. Kippenberg, S. M. Spillane, and K. J. Vahala ${ }^{a)}$ \\ Thomas J. Watson Laboratory of Applied Physics, California Institute of Technology, Pasadena, \\ California 91125
}

(Received 12 July 2004; accepted 16 October 2004)

\begin{abstract}
Optical microcavities confine light spatially and temporally and find application in a wide range of fundamental and applied studies. In many areas, the microcavity figure of merit is not only determined by photon lifetime (or the equivalent quality-factor, Q), but also by simultaneous achievement of small mode volume (V). Here we demonstrate ultra-high Q-factor small mode volume toroid microcavities on-a-chip, which exhibit a Q/V factor of more than $10^{6}(\lambda / n)^{-3}$. These values are the highest reported to date for any chip-based microcavity. A corresponding Purcell factor in excess of 200000 and a cavity finesse of $>2.8 \times 10^{6}$ is achieved, demonstrating that toroid microcavities are promising candidates for studies of the Purcell effect, cavity QED or biochemical sensing. (C) 2004 American Institute of Physics. [DOI: 10.1063/1.1833556]
\end{abstract}

Microcavities can be characterized by two figures of merits: the temporal confinement is described by the quality factor of the mode $(\mathrm{Q})$, and the spatial confinement is characterized by the mode volume $(\mathrm{V}) .{ }^{1}$ Of all optical microcavities, whispering-gallery-type microsphere resonators have obtained the highest $\mathrm{Q}$ factor to date (nearly 9 billion $^{2}$ ). While Q-factor figures prominently in many applications, minimizing mode volume is also important in a variety of fundamental and applied studies such as cavity quantum electrodynamics (cQED) studies, photonics and biochemical sensing. In particular, a high $\mathrm{Q} / \mathrm{V}$ ratio is desirable in applications such as lasers, add-drop filters and biochemical sensors, which rely upon a large finesse. Cavities can also be used to enhance the spontaneous emission rate, a concept which is used in "single-photon on-demand" sources, where the figure of merit is the Purcell factor, given by

$$
F_{p}=\frac{3}{4 \pi^{2}} \frac{Q}{V}\left(\frac{\lambda}{n}\right)^{3}
$$

(Ref. 5). Wafer-based cavities such as photonic crystals, ${ }^{6,7}$ microposts, ${ }^{3}$ or microdisks ${ }^{8}$ typically have much smaller mode volume than microspheres, and allow wafer-scale integration and control. ${ }^{9}$ However, the Q factor of these cavities has remained significantly lower than for silica microspheres. However, recently, ultra-high-Q performance on a chip has been demonstrated using a toroid-microcavity. ${ }^{10}$ These cavities allow integration and control previously not accessible in the ultra-high-Q regime. Here we demonstrate that toroid microcavities not only allow one to obtain ultrahigh-Q, but also to achieve small mode volumes, reaching a previously inaccessible range of $\mathrm{Q} / \mathrm{V}$ ratio of more than $10^{6}(\lambda / n)^{-3}$. By variations of the principal and minor toroid diameter, the $\mathrm{Q} / \mathrm{V}$ value was adjusted and the highest achieved value was $Q / V_{m} \approx 2.5 \times 10^{6}(\lambda / n)^{-3}$ (for a toroid microcavity with $\lambda=1550 \mathrm{~nm}, V_{m} \approx 180 \mu \mathrm{m}^{3}$, and $Q \approx 4$ $\times 10^{8}$ ). This result is more than one order of magnitude larger than the highest value reported so far, using photonic

\footnotetext{
${ }^{a)}$ Electronic mail: vahala@its.caltech.edu
}

crystal defect cavities. ${ }^{6}$ Further optimization of the toroid microcavity can result in yet higher values.

The use of silica microtoroids allows the preservation of ultra-high-Q factors while the additional transverse spatial confinement of the optical mode over spherical cavities results in a smaller modal volume. To investigate the modal volumes of toroid microcavities achievable experimentally, samples were fabricated as described in Ref. 10, by lithography and etching followed by a $\mathrm{CO}_{2}$ laser-assisted reflow process. Figure 1 shows a scanning electron microscopy image of a toroid microcavity. The geometrical parameters defining the structure are the minor toroid diameter $(d)$ and the principal toroid diameter (denoted $D$ ). The principal toroid diameter $(D)$ was controlled by the size of the initial silica disk preform. The thickness of the toroid (i.e., minor diameter $d$ ) is determined by a combination of the initial oxide thickness and the flux and exposure time of the subsequent $\mathrm{CO}_{2}$ laser anneal. In order to create small mode volume microcavities, $1 \mu \mathrm{m}$ thermally oxidized silicon wafers were used. Subsequently, photolithography and etching were used to create various diameter silica disk preforms, with the smallest diameter being $20 \mu \mathrm{m}$. The laser assisted reflow was used in a final step to create toroidal microcavities. During this process the silica melts, and the toroid principal diameter is reduced while silica is consumed to form the toroidal pe-

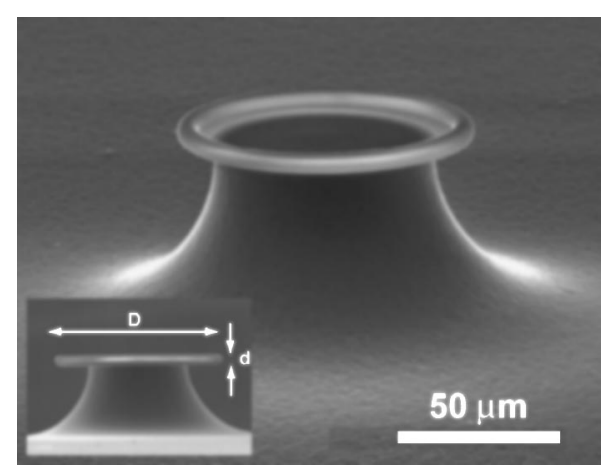

FIG. 1. Scanning-electron-microscopy image of a high $\mathrm{Q} / \mathrm{V}$ toroid microcavity on a chip, using a $1 \mu \mathrm{m}$ thermal oxide microdisk as a preform. Inset: the principal (D) and minor (d) toroid diameter. 


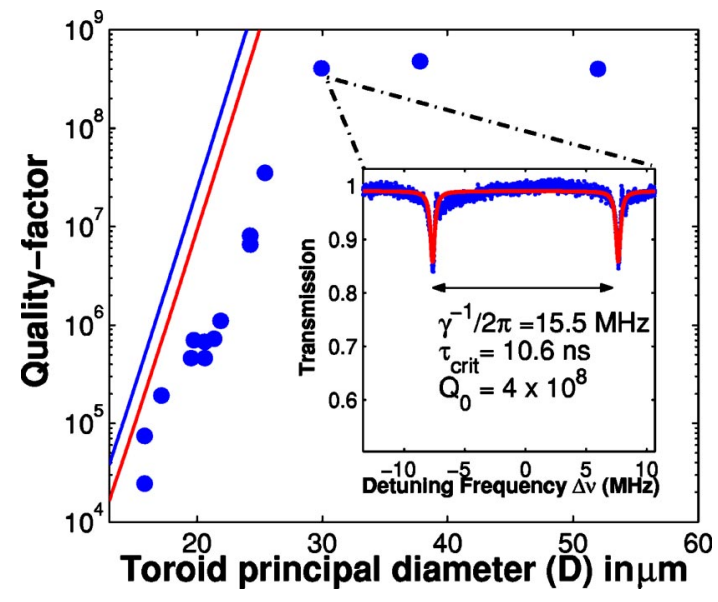

FIG. 2. Measured quality factor of toroid microcavities, with approximately constant minor diameter $(d=3.5 \mu \mathrm{m})$ and varying principal diameter. The solid lines are the theoretically predicted whispering-gallery quality factor for a microsphere (TE, blue), (TM, red). Inset: The doublet resonance structure for a $28-\mu \mathrm{m}$-diameter toroid microcavity. The critical decay time measured for this sample was $10.6 \mathrm{~ns}$. The intrinsic Q-factor is $4 \times 10^{8}$, corresponding to a modal coupling parameter of $\Gamma=31$. Solid line is a fit to a coupled oscillator model (Ref. 14).

riphery. For a 1- $\mu \mathrm{m}$-thick thermal oxide, the smallest toroidal cross sections (minor) diameters obtained in this work were $\sim 3.3 \mu \mathrm{m}$ and principal diameters as small as $12 \mu \mathrm{m}$ were achieved, as inferred by scanning-electron microscopy. It should be noted that for oxide layers below $1 \mu \mathrm{m}$, it was found that the residual strain in the oxide caused nonuniform silica disk preforms.

Characterization of the quality factor of microtoroids proceeded by coupling the optical whispering-gallery modes to tapered optical fibers. A piezoelectric three-axis stage with $20 \mathrm{~nm}$ closed-loop resolution allowed accurate control of the taper-microtoroid coupling gap. Tapers with diameters of $\sim 1-2 \mu \mathrm{m}$ were used, and efficient coupling with high ideality was achieved. ${ }^{11}$ A narrow line-width external cavity laser was used to excite the optical modes, and the taper transmission recorded. The ultra-high-Q toroid modes typically exhibited a doublet structure due to the presence of scattering centers which can induce modal coupling of the degenerate clockwise and counterclockwise whispering-gallery modes. ${ }^{12,13}$ The strength of intermode coupling (i.e., visibility of the doublet structure, as given by the ratio of the cavity linewidth $\tau^{-1} / 2 \pi$ and the splitting frequency $\gamma^{-1} / 2 \pi$ ) can be described by the dimensionless modal coupling parameter $\Gamma \equiv \tau_{0} / \gamma,{ }^{13}$ and was found to generally increase for decreasing microcavity size, due to the enhanced scattering-capture efficiency of the WGM modes. The inset of Fig. 2 shows the doublet structure of a WGM in a $28-\mu \mathrm{m}$-diameter toroid (with the doublet splitting frequency $\gamma / 2 \pi=15.5 \mathrm{MHz}$, and $\Gamma=31$ ). In addition, the optical modes exhibited strong thermal effects even at low powers, due to their small mode volume. These thermal effects induce distortion of the lineshape unless input power levels are adjusted to a suitably low value. These distortions tend to make the apparent linewidth larger (pull the resonant frequencies of the mode) in one frequency scan direction while making its appearance narrower in the opposing scan direction. These artifacts present a challenge to $\mathrm{Q}$ measurement based upon linewidth measurement alone. In order to accurately determine the $\mathrm{Q}$ in presence of strong modal coupling and thermal effects, an Downloaded 17 Dec 2006 to 131.215.240.9. Redistribution subject alternative approach was used. This approach does not rely upon measurement of cavity linewidth, but on measurement of the doublet splitting frequency $\left(\gamma^{-1} / 2 \pi\right)$ and the cavity ringdown time at the critical coupling point. ${ }^{14}$ Alternatively, the cavity $\mathrm{Q}$ can be inferred by measurement of the modal coupling parameter $\Gamma$ and a ringdown measurement, as is presented in Ref. 10.

In comparison with the latter, the method presented here does not require knowledge of $\Gamma,{ }^{13}$ but rather only the doublet splitting frequency $\left(\gamma^{-1} / 2 \pi\right)$. Measurement of splitting frequency is less sensitive to thermal effects since the splitting frequency has been observed to be nearly immune to thermal shifts (assuming that each mode is frequency shifted nearly equally by the excitation wave). Therefore, the splitting frequency can be deduced even in a regime where the individual doublets are thermally distorted. To infer the quality factor, the measurement of the splitting frequency is combined as in previous studies, with the ring-down lifetime at the critical coupling point $\left(\tau_{\text {crit }}\right)$. The longest critical decaytime observed in this study was $\tau_{\text {crit }}=75 \mathrm{~ns}$. The following expression is then used to relate both this information and the measured splitting frequency to the intrinsic $\mathrm{Q}$ factor:

$$
Q_{0}=\omega \tau_{0}=\omega \frac{2}{\tau_{\text {crit }}}\left(\frac{1}{\tau_{\text {crit }}^{2}}-\frac{1}{\gamma^{2}}\right)^{-1}
$$

Figure 2 shows the quality factor determined through this method for a number of microtoroids as a function of toroid principal diameter $(D)$ for minor diameter in the range of $(d=2.8-6 \mu \mathrm{m})$. The highest measured value was $4 \times 10^{8}$, in a toroid microcavity with geometrical parameters $d=6 \mu \mathrm{m}$, $D=29 \mu \mathrm{m}$, and $\lambda \approx 1550 \mathrm{~nm}$. As evident from the experimental results, ultra-high-Q $\left(>10^{8}\right)$ factor could be maintained until a limiting diameter of $\sim 28 \mu \mathrm{m}$, below which point a strong decrease of $\mathrm{Q}$ factor is observed. Reduction of $\mathrm{Q}$ for smaller principal diameters is expected, as the whispering-gallery loss increases as a result of the increased optical leakage through the whispering-gallery potential. ${ }^{15,16}$ In a sphere the whispering-gallery $\mathrm{Q}$ scales as $Q \propto \exp (2 \ell)$, where $\ell$ is the angular mode number. ${ }^{16,17}$ Numerical modeling confirms that the angular mode number $\ell$ for the measured microtoroids is nearly unchanged compared to that of a microsphere of identical principal diameter, and the radial eigenfunctions are still well described by their microsphere counterparts. Therefore, the Q-factor is expected to approximately scale according to the whispering-gallery loss of a microsphere. For comparison, the numerically calculated whispering-gallery-loss for a microsphere TE(TM) mode is also shown in Fig. 2 and tracks the measured Q toroid Q values in the leaky regime. Also evident from Fig. 2, the toroid microcavities of this work nonetheless show Q-values typically more than 1 order of magnitude below the expected whispering-gallery-loss limit for a spherical cavity, indicating that other contributing factors such as surface scattering and surface contaminant absorption also increase with decreasing diameter. This is consistent with observations in silica microspheres. $^{2}$

To infer the mode volume of the fundamental whispering-gallery modes of the microcavities, we used a numerical finite-element modeling method in conjunction with the exact toroid geometry (as determined from scanning-electron microscopy) to solve for the optical modes to AIP license or copyright, see http://apl.aip.org/apl/copyright.jsp 




FIG. 3. Ratio of Q-factor and mode volume (V) using the experimental data from Fig. 2. The mode volume was estimated by numerical finite element modeling using the cavity geometry parameters, as inferred by SEM. Dotted lines give the asymptotic behavior. For large diameter, the intrinsic $Q$ is approximately constant, and the $\mathrm{Q} / \mathrm{V}$ ratio follows an inverse square dependence on principal cavity diameter, whereas for small diameters the exponential dependence of $Q$ on principal diameter dominates. Inset: The mode area of a toroid microcavity as a function of minor toroid diameter (d) for fixed principal toroid diameter $(D=25,50$, and $75 \mu \mathrm{m}$ are shown). The corresponding mode volume is given by $\mathrm{V}=\pi D A_{\text {eff }}$. The highest $\mathrm{Q} / \mathrm{V}$ value of more than $10^{6}(\lambda / n)^{-3}$ was measured for a $29-\mu$ m-principal-diameter toroid.

of the toroid. Mode volume can then be evaluated from the commonly used definition (where $\varepsilon$ is the dielectric constant):

$$
V=\frac{\int \varepsilon|\mathbf{E}(r)|^{2} d V}{\max \left[\varepsilon|\mathbf{E}(r)|^{2}\right]} .
$$

The inset of Fig. 3 shows the mode volume of the fundamental TE whispering-gallery modes, as a function of minor toroid diameter $d$ (for a principal diameter of 25, 50, and 75 $\mu \mathrm{m})$. As expected, the mode volume is continuously reduced as a function of both decreasing principal diameter (D) and minor diameter $(d)$. For a fixed principal diameter D, reduction of minor diameter causes first a slow reduction of mode volume [scaling as $(d / D)^{1 / 4}$, due to azimuthal compression of the mode], followed by a strong compression of the mode in both radial and azimuthal directions. Figure 3 shows the $\mathrm{Q} / \mathrm{V}$ ratio determined for the samples under consideration in this study. The $\mathrm{Q} / \mathrm{V}$ value exhibits an optimum, which occurs near a principal diameter of $\sim 29 \mu \mathrm{m}$. At large principal diameters the intrinsic quality factor is experimentally found to be nearly independent of radius (believed to be limited by scattering and $\mathrm{OH}$ absorption contributions) and for this reason reduction of the principal cavity diameter allows an increase in $\mathrm{Q} / \mathrm{V}$ ratio. However, the $\mathrm{Q} / \mathrm{V}$ value cannot be increased indefinitely, as the reduction of mode volume ultimately comes at the expense of reduced quality factor due to the presence of tunneling loss. For radii where the tunnel loss becomes the dominant loss mechanism, the reduction in mode volume is more than offset by a much more strongly reduced quality factor, adversely affecting the $\mathrm{Q} / \mathrm{V}$ value. Therefore, for a given minor diameter $(d)$, an optimum $\mathrm{Q} / \mathrm{V}$ value exists. More generally, since the mode volume (and Q) depends on both minor and outer toroid diameter, there is also an absolute maximum of the $\mathrm{Q} / \mathrm{V}$ value for toroid mi- crocavities. Numerical modeling of the maximum achievable $\mathrm{Q} / \mathrm{V}$ for a toroidal geometry was also investigated, and will be presented elsewhere.

The maximum achievable $\mathrm{Q} / \mathrm{V}$ factor in these studies was $2.5 \times 10^{6}(\lambda / n)^{-3}$, for a microtoroid with $D=29 \mu \mathrm{m}, d$ $=6 \mu \mathrm{m}, Q=4 \times 10^{8}, V_{m} \approx 180 \mu \mathrm{m}^{3}$ at $\lambda=1550 \mathrm{~nm}$. The highest reported $\mathrm{Q} / \mathrm{V}$ factor to date for an optical microcavity is $4.5 \times 10^{4}(\lambda / n)^{3}$ for a photonic crystal defect cavity. ${ }^{6}$ Therefore, the measured value in this work constitutes a more than one order of magnitude improvement, and enters an unprecedented $\mathrm{Q} / \mathrm{V}$ range of more than $10^{6}(\lambda / n)^{-3}$. To allow further comparison with other microcavity geometries, the Purcell factor and finesse are also calculated. The cavity-finesse $\left(>2.8 \times 10^{6}\right)$ exceeds the highest value reported so far of $2.3 \times 10^{6}{ }^{2}$ In addition a Purcell-factor of $2 \times 10^{5}$ is achieved, which is an improvement of more than one order of magnitude over previously reported values. ${ }^{3,6,7}$

In summary, ultra-high-Q small mode volume toroid microcavities are demonstrated. By reduction of the toroidal cross section and variation of the cavity principal diameter an optimum ratio of quality factor to mode volume $(\mathrm{Q} / \mathrm{V})$ was obtained. The mode volume reduction was calculated using numerical modeling of the toroid modes. The highest achieved $\mathrm{Q} / \mathrm{V}$ value was in excess of $10^{6}(\lambda / n)^{-3}$ and is more than one order of magnitude larger than for any other chipbased microcavity. Corresponding Purcell factor and cavity finesse are also record values in this device. The large Purcell-factor shows that toroid microcavities are promising candidates for achieving enhancement of spontaneous emission, and related studies.

This work was funded by the DARPA, NSF, and the Caltech Lee Center for Advanced Networking.

${ }^{1}$ K. J. Vahala, Nature (London) 424, 839 (2003).

${ }^{2}$ D. W. Vernooy, V. S. Ilchenko, H. Mabuchi, E. W. Streed, and H. J. Kimble, Opt. Lett. 23, 247 (1998).

${ }^{3}$ J. M. Gerard, B. Sermage, B. Gayral, B. Legrand, E. Costard, and V. Thierry-Mieg, Phys. Rev. Lett. 81, 1110 (1998).

${ }^{4}$ E. Moreau, I. Robert, J. M. Gerard, I. Abram, L. Manin, and V. ThierryMieg, Appl. Phys. Lett. 79, 2865 (2001).

${ }^{5}$ E. M. Purcell, Phys. Rev. 69, 681 (1946)

${ }^{6}$ Y. Akahane, T. Asano, B. S. Song, and S. Noda, Nature (London) 425, 944 (2003).

${ }^{7}$ K. Srinivasan, P. E. Barclay, M. Borselli, and O. Painter, Phys. Rev. B 70, 081306 (2004)

${ }^{8}$ B. Gayral, J. M. Gerard, A. Lemaitre, C. Dupuis, L. Manin, and J. L. Pelouard, Appl. Phys. Lett. 75, 1908 (1999); A. Kiraz, P. Michler, C. Becher, B. Gayral, A. Imamoglu, L.. Zhang, E. Hu, W. V. Schoenfeld, and P. M. Petroff, ibid. 78, 3932 (2001).

${ }^{9}$ P. Lalanne and J. P. Hugonin, IEEE J. Quantum Electron. 39, 1430 (2003).

${ }^{10}$ D. K. Armani, T. J. Kippenberg, S. M. Spillane, and K. J. Vahala, Nature (London) 421, 925 (2003).

${ }^{11}$ S. M. Spillane, T. J. Kippenberg, O. J. Painter, and K. J. Vahala, Phys. Rev. Lett. 91, 043902 (2003).

${ }^{12}$ D. S. Weiss, V. Sandoghdar, J. Hare, V. Lefevreseguin, J. M. Raimond, and S. Haroche, Opt. Lett. 20, 1835 (1995).

${ }^{13}$ T. J. Kippenberg, S. M. Spillane, and K. J. Vahala, Opt. Lett. 27, 1669 (2002).

${ }^{14}$ M. Cai, O. Painter, and K. J. Vahala, Phys. Rev. Lett. 85, 74 (2000).

${ }^{15}$ R. K. Chang and A. J. Campillo, Optical Processes in Microcavities (World Scientific, Singapore, 1996).

${ }^{16} \mathrm{~L}$. Weinstein, Open Resonators and Open Waveguides (Golem, Boulder, Co, 1996).

${ }^{17}$ V. V. Datsyuk, Appl. Phys. B: Photophys. Laser Chem. 54, 184 (1992). 